\title{
Safety Analysis of 10 Clinical Trials and for 13 Years After First Approval of Ioflupane ${ }^{123}$ I Injection (DaTscan)
}

\author{
Donald G. Grosset*1, Klaus Tatsch ${ }^{2}$, Wolfgang H. Oertel ${ }^{3}$, Eduardo Tolosa ${ }^{4}$, Nin Bajaj ${ }^{5}$, Andreas Kupsch ${ }^{6}$, \\ John T. O’Brien ${ }^{7}$, John Seibyl ${ }^{8}$, Zuzana Walker ${ }^{9,10}$, Paul Sherwin ${ }^{11}$, Chris Chen ${ }^{12}$, and Igor D. Grachev*11 \\ ${ }^{l}$ Institute of Neurological Sciences, Department of Neurology, Southern General Hospital, University of Glasgow, Glasgow, Scotland, \\ United Kingdom; ${ }^{2}$ Department of Nuclear Medicine, Municipal Hospital Karlsruhe, Inc., Karlsruhe, Germany; ${ }^{3}$ Department of \\ Neurology, Philipps-University of Marburg, Marburg, Germany; ${ }^{4}$ Neurology Service, Hospital Clinic de Barcelona, Universitat de \\ Barcelona, IDIBAPS, Centro de Investigación Biomédica en Red sobre Enfermedades Neurodegenerativas (CIBERNED), Barcelona, \\ Catalonia, Spain; ${ }^{5}$ Department of Clinical Neurology, Nottingham University Hospitals NHS Trust, Nottingham, United Kingdom; \\ ${ }^{6}$ Department of Neurology and Stereotactic Neurosurgery, Otto-von-Guericke-University, Magdeburg, Germany; ${ }^{7}$ Department of \\ Psychiatry, University of Cambridge, and Cambridgeshire and Peterborough Foundation NHS Foundation Trust, Cambridge, United \\ Kingdom; ${ }^{8}$ The Institute for Neurodegenerative Disorders, New Haven, Connecticut; ${ }^{9}$ Mental Health Sciences Unit, University \\ College London, Bloomsbury Campus, London, United Kingdom; ${ }^{10}$ North Essex Partnership NHS Foundation Trust, Essex, United \\ Kingdom; ${ }^{11}$ Life Sciences, GE Healthcare, Princeton, New Jersey; and ${ }^{12} \mathrm{H} 2 \mathrm{O}$ Clinical, LLC, Hunt Valley, Maryland
}

loflupane is an analog of cocaine that binds reversibly with high affinity to the dopamine transporter (DaT) protein, a marker for presynaptic terminals in dopaminergic nigrostriatal neurons. loflupane ${ }^{123}$ I Injection is also known as DaTscan or DaTSCAN (123/-ioflupane is also called ${ }^{123} \mid-2-\beta$-carbomethoxy-3 $\beta$-(4iodophenyl)- $N$-(3-fluoropropyl)nortropane or ${ }^{123}$ I-FP-CIT). The diagnostic efficacy of DaTscan has been described elsewhere. Here, we present a comprehensive analysis of the safety of DaTscan starting from initiation of clinical development through $13 \mathrm{y}$ after the date of first market approval. Safety data in the sponsor's clinical development safety database from 10 completed DaTscan clinical trials were pooled, and postapproval experience was summarized from standardized aggregate safety reports submitted to regulatory agencies. A total of 1,180 clinical trial subjects $(92 \%$ of 1,284 subjects planned to receive DaTscan in the clinical trials) received DaTscan. Percentages of subjects with adverse events by category were as follows: all (22\%), considered at least possibly related to DaTscan by the investigator (4\%), any severe (3\%), headache $(4 \%)$, nausea $(2 \%)$, dizziness $(2 \%)$, nasopharyngitis $(1 \%)$, and injection site hematoma (1\%). Four percent of subjects had at least 1 serious adverse event; 5 subjects $(<1 \%)$ had serious adverse events that led to death. All serious adverse events, including those that led to death, were deemed by an expert clinician to be unrelated to DaTscan. An estimated half a million market doses of DaTscan (for single use) were administered from July 2000 through the July 2013 reporting period. In postapproval safety assessment, 1 death was reported $20 \mathrm{~d}$ after (and unrelated to) DaTscan administration. Two spontaneously reported serious adverse drug reactions (ADRs) and 32 spontaneously reported nonserious ADRs were submitted, approximately half of which are identified in labeling. Headache (in clinical trials) and injection site pain (postapproval) were the most commonly reported events or reactions. Although adverse events were reported for 1 in 5 clinical trial

Received Jan. 27, 2014; revision accepted Apr. 7, 2014.

For correspondence or reprints contact: Igor Grachev, GE Healthcare, 101 Carnegie Center, Princeton, NJ 08540.

E-mail: grachevi@hotmail.com

${ }^{*}$ Contributed equally to this work.

Published online Jun. 19, 2014.

COPYRIGHT (c) 2014 by the Society of Nuclear Medicine and Molecular Imaging, Inc. subjects, most were mild and considered unrelated to DaTscan administration. Severe events were uncommon, and no serious adverse event occurring in more than 1 subject was deemed related to DaTscan administration. In postapproval experience, the frequency of ADRs spontaneously reported was less than 1 per 10,000 doses administered. Comprehensive safety data show that DaTscan was well tolerated.

Key Words: clinical trials systematic review/meta-analysis; dementia with Lewy bodies; Parkinson disease/parkinsonism; patient safety; SPECT

J Nucl Med 2014; 55:1281-1287

DOI: 10.2967/jnumed.114.138032

I oflupane is an analog of cocaine that binds reversibly with high affinity to the dopamine transporter (DaT) protein, a marker for presynaptic terminals in dopaminergic nigrostriatal neurons. Ioflupane ${ }^{123}$ I Injection (GE Healthcare), also known as DaTscan or DaTSCAN, contains both radioactive ( ${ }^{123} \mathrm{I}$-ioflupane also called ${ }^{123}$ I-2- $\beta$-carbomethoxy-3 $\beta$-(4-iodophenyl)- $N$-(3-fluoropropyl)nortropane or ${ }^{123}$ I-FP-CIT) and nonradioactive ioflupane, which occupy less than $1 \%$ of DaT protein binding sites in the brain at a mass dose of $0.325 \mu \mathrm{g}$ or less. For reference, about $60 \%$ occupancy is needed for cocainelike effects (1). ${ }^{123}$ I radioactive decay releases $\gamma$ radiation, allowing visualization of the striata through SPECT imaging.

Ioflupane ${ }^{123}$ I Injection was first approved as DaTSCAN by the European Agency for the Evaluation of Medicinal Products (now European Medicines Agency) on July 27, 2000. In addition to its authorization in the European Union (EU) via the centralized procedure, it is also approved in Israel, Switzerland, and the United States (as DaTscan, approved January 14, 2011) (a total of 33 countries).

In the United States, DaTscan is approved as an adjunct to other diagnostic evaluations in the assessment of adult patients with suspected parkinsonian syndromes (PS) to help differentiate essential tremor (ET) from tremor due to PS (idiopathic Parkinson 
disease [PD], multiple system atrophy [MSA], and progressive supranuclear palsy [PSP]) (2). In the EU, DaTscan is approved for detecting loss of DaT in patients with clinically uncertain or early (added in 2013) PS to help differentiate ET from PS related to PD, MSA, and PSP and probable dementia with Lewy bodies (DLB) from Alzheimer disease (AD) (added in 2006) (3). DaTscan is unable to discriminate between PD, MSA, and PSP or DLB from PD dementia. The diagnostic efficacy of DaTscan has been described elsewhere (4-9).

Here, we present a comprehensive analysis of the safety of DaTscan. Data were collected during clinical development of DaTscan from 10 completed clinical trials, clinical conduct for the first of which began on April 25, 1996, and the latest of which ended on October 8, 2012, and during postapproval surveillance from July 27, 2000 (first approval) until July 27, 2013. Safety surveillance data were summarized from standardized aggregate safety reports submitted to regulatory agencies.

\section{MATERIALS AND METHODS}

\section{Clinical Trials}

Details by trial are summarized in Supplemental Table 1 (supplemental materials are available at http://jnm.snmjournals.org). All 10 trials were sponsored by GE Healthcare. All trials were conducted according to International Conference on Harmonisation (ICH) Good Clinical Practice Guidelines.

All trials were formally approved by human ethics committees; each subject in every trial or their legal guardian (according to local regulations) signed a written informed consent form. Studies PDT301 and PDT409 are identified in clinicaltrials.gov as NCT00209456 and NCT00382967, respectively. PDT409 and GE001-013 are identified with EU Drug Regulatory Affairs Clinical Trials (EudraCT) numbers 2005-002216-25 and 2010-021474-11, respectively. All other trials began enrolling before July 1, 2005, the cutoff date for the initiation of the requirement by the International Committee of Medical Journal Editors for trials to be registered, so are not associated with any public database identifiers.

In addition to adult male and female healthy volunteers (HVs), patients with the following clinical diagnoses were enrolled: PS (PD, MSA, PSP), clinically uncertain PS, DLB (possible and probable), non-PS; ET; and dementia (e.g., AD, vascular dementia). All trials were conducted in the EU except PDT409, which also enrolled patients in the United States. Each subject received a baseline clinical diagnosis, based whenever possible on validated or currently accepted consensus criteria (10) as shown in Supplemental Table 2. The patient population by baseline diagnosis and the main purpose of each study, for example, biodistribution, uptake kinetics, safety and tolerability, diagnostic efficacy, or clinical utility, are indicated in Supplemental Table 1 and its footnotes.

Use of any concomitant medication that was known or suspected (at the time of the trial) to interact with striatal uptake through direct competition with binding of ioflupane to DaT was excluded. Potentially interfering medications included amphetamine, benztropine, bupropion, cocaine, mazindol, methylphenidate, phentermine, and sertraline.

Women who were breastfeeding or pregnant were excluded. Subjects with significant renal or hepatic impairment, for example, serum creatinine or hepatic transaminase greater than 3 times the upper limit of normal (ULN), bilirubin greater than 2 times the ULN, $\gamma$-glutamyl transferase greater than 5 times the ULN, or abnormal prothrombin time or creatinine phosphokinase isoenzymes (myocardial bands and myocardial muscle fractions) were excluded. Cancer other than cerebral tumor was not an explicit exclusion criterion in any study.
The 10 open-label, completed clinical trials comprised phase 1 (1), phase 2 (2), phase 3 (4), phase 3b/4 (1), and phase 4 (2). All trials after phase 2 were multicenter. The trials, which included subjects with non-PS (PDT02005, DP008-003, PDT304, and PDT03007) as well as the trials with a group not receiving DaTscan (PDT409 and GE-001013), were considered controlled. The 2 phase- 4 trials were both randomized and controlled. Trials were single dose with the following exceptions: PDT304 (up to 3 doses at 18-mo intervals), PDT408 (up to 2 doses, 24 mo apart), and subjects in PDT03007 were from DP008003 and received up to 2 doses (1 dose in each study). PDT409 and GE-001-013, although single-dose trials, incorporated a 1-y and 6-mo follow-up visit, respectively.

Treatment-emergent adverse events (AEs), including laboratory abnormalities, were monitored from the start of each injection for generally 2-7 d after each dose (longer if necessary) in the phase 1 through 3 trials. In the phase $3 \mathrm{~b} / 4$ trial and 2 phase- 4 trials, safety events were monitored between start of injection and end of scan and again at the discrete follow-up time point. AEs were classified by preferred term according to the Medical Dictionary for Regulatory Activities [MedDRA] (version 11.0 for 8 studies (11), version 13.1 for PDT409 (12), and version 15.0 for GE-001-013 (13)).

A battery of safety tests was performed including laboratory evaluations, vital signs, and electrocardiograms before and after dosing, as detailed by trial in Supplemental Table 3. Concomitant medication, defined as all medications taken while receiving DaTscan or taken after the first dose of DaTscan in 9 studies and all medications reported in PDT301 (because start date was inadvertently not collected), were classified according to the World Health Organization (WHO) Anatomic Therapeutic Chemical Classification System.

\section{Postapproval Surveillance}

According to ICH Guideline E2D (November 12, 2003), a reaction, in contrast to an event, is characterized by the fact that a causal relationship between the drug and the occurrence is suspected. For regulatory reporting purposes, however, if an event is spontaneously reported, even if the relationship is unknown or unstated, it meets the definition of an ADR. Postapproval surveillance data included all ADRs spontaneously reported by patients or physicians for the period July 27, 2000, through July 27, 2013 (before adoption of ICH E2D, occurrences were called spontaneous reports). ADRs included spontaneous reports to the manufacturing authorization holder (GE Healthcare) and from investigator-initiated studies (758 subjects in 32 studies comprising EU member states, the United States, and Israel).

\section{DaTscan Administration and SPECT}

At least $1 \mathrm{~h}$ before administration of DaTscan (Ioflupane ${ }^{123} \mathrm{I}$ Injection), for protection of the thyroid from ${ }^{123}$ I uptake, thyroid blocking agents were to be administered according to protocol (phase 1 through 3 trials) or DaTscan label and hospital routine (phase-4 trials and postapproval surveillance). DaTscan was administered intravenously and followed by a $5-\mathrm{mL}$ saline flush (clinical trials). SPECT scanning was performed 3-6 h after DaTscan injection. A comparable dose of DaTscan was used in all studies: $111-185 \mathrm{MBq}(3-5 \mathrm{mCi})$. DaTscan dosing is based on the radioactivity determined using a suitably calibrated instrument immediately before administration. The marketed recommended dose is $111-185 \mathrm{MBq}$.

\section{Statistical Considerations}

For clinical trials, data from the database for the Integrated Summary of Safety from the U.S. New Drug Application (NDA) comprising 8 studies, plus data from 2 completed postapproval studies, GE-001-013 and PDT409 (only the subjects who received DaTscan), were pooled and summarized using SAS (SAS Institute Inc.). 
The total number of AEs is reported. Descriptive statistics are provided for the number of subjects with at least $1 \mathrm{AE}$ and the number of subjects with at least $1 \mathrm{AE}$ leading to discontinuation from study, leading to death, and that was a serious $\mathrm{AE}$ (defined as any $\mathrm{AE}$ that resulted in death, was life-threatening, required inpatient hospitalization or prolongation of existing hospitalization, resulted in persistent or significant disability or incapacity, was a congenital anomaly or birth defect, or was another important medical event that could jeopardize the subject and might have required medical intervention to prevent one of the aforementioned outcomes). AEs occurring in more than 1 subject were manually compiled from the SAS output. AEs (including serious AEs) were categorized by maximum intensity (i.e., mild, moderate, or severe) and maximum relatedness (i.e., at least possibly related) to DaTscan administration according to the investigator's judgment. The results summaries are not mutually exclusive: it is possible for a single specific event to fall under all of the following descriptors-all AEs, AEs leading to discontinuation from the study, serious AEs, related AEs, and severe AEs. Subgroups of sex and age $(<65 \mathrm{y}, \geq 65 \mathrm{y}$ and $<75 \mathrm{y}, \geq 75 \mathrm{y})$ are provided for each $\mathrm{AE}$ tabulation.

Postapproval surveillance data from standardized aggregate safety reports submitted to regulatory agencies are summarized separately.

\section{RESULTS}

\section{Disposition, Demographics, and Other Relevant Subject Characteristics}

Subjects enrolled in the study totaled 1,$482 ; 1,284$ subjects were randomized or assigned to DaTscan and 198 subjects as controls (no DaTscan) (Fig. 1). One hundred four subjects discontinued before receiving DaTscan, and 1,180 subjects (92\% [1,180/ 1,284]) received DaTscan as planned (safety population). One hundred two subjects $(9 \%)$ discontinued after at least 1 dose of DaTscan, including $14(1 \%)$ for safety reasons. Discontinuation could have been a reflection of the length of the trial, as most discontinuations (80\%) came from Study PDT304, the 3-y study (most from 3 to $18 \mathrm{mo}$ after the first dose and before the second dose).

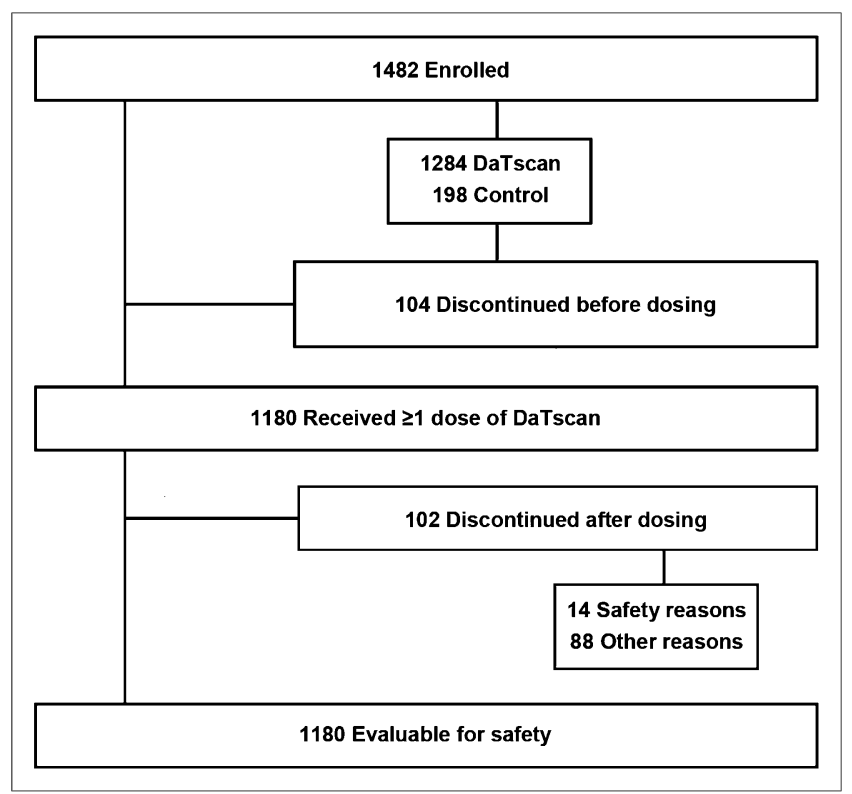

FIGURE 1. Disposition of subjects in 10 completed DaTscan trials.
TABLE 1

Overall Demographic Characteristics and Final Diagnoses of Safety Evaluable Subjects in 10 Completed DaTscan Clinical Trials

\begin{tabular}{|cc}
\hline \multicolumn{1}{c}{ Characteristic } & DaTscan $(n=1,180)$ \\
\hline Sex $(n)$ & $670(57)$ \\
\hline Male & $506(43)$ \\
\hline Female & $4(<1)$ \\
\hline Missing & \\
\hline Age $(\mathrm{y})$ & $67.3 \pm 11.08$ \\
Mean \pm SD & $418(35)$ \\
$<65(n)$ & $758(64)$ \\
$\geq 65(n)$ & $4(<1)$ \\
Missing $(n)$ & $833(71)$ \\
\hline$<75(n)$ & $343(29)$ \\
\hline$\geq 75(n)$ & $4(<1)$ \\
Missing $(n)$ & $73.0(13.76)$ \\
Mean weight \pm SD $(\mathrm{kg})$ & $25.7(4.22)$ \\
Mean body mass index \pm SD $\left(\mathrm{kg} / \mathrm{m}^{2}\right)$ & \\
Diagnosis $(n)$ & $456(38.6)$ \\
\hline PS & $242(20.5)$ \\
DLB & $49(4.2)$ \\
\hline ET & $57(4.8)$ \\
\hline Healthy volunteer & $330(28)$ \\
Other & $46(3.9)$ \\
\hline Unknown & \\
\hline
\end{tabular}

*For example, $A D$, vascular dementia.

Data in parentheses are percentages.

The study population was $57 \%$ male, $64 \%$ of subjects were $65 \mathrm{y}$ or older, $29 \%$ of subjects were 75 y or older (Table 1), and $97 \%$ of all subjects were from the EU (only 35 subjects from PDT409 were from the United States). Supplemental Table 4 lists numbers of subjects by final diagnosis and trial. Most subjects (1,008 [85\%]) received exactly 1 dose of DaTscan. Seventy-three subjects (6\%) received exactly 2 doses, and 99 subjects $(8 \%)$ received exactly 3 doses. Overall, mean activity administered was $167 \mathrm{MBq}$ per injection (highest for DLB [182 MBq] and lowest for HV [138 MBq]).

All non-HVs (100\%) and $67 \%$ of HVs took concomitant medication. The most frequently used concomitant medications fell into WHO primary classes as follows: systemic hormonal preparations excluding sex hormones and insulin $(85 \%$ [84\% thyroid-blocking medications]), nervous system (80\%), and cardiovascular system (54\%). The most frequently used concomitant medications by diagnosis category and WHO secondary class were as follows: $74 \%$ of PS subjects took antiparkinson drugs, $79 \%$ of DLB subjects took psychoanaleptics, $31 \%$ of ET subjects took antithrombotic agent, $31 \%$ of ET subjects took antiepileptics, $9 \%$ of HVs took sex hormones and modulators of the genital system, $60 \%$ of subjects with other diagnoses took psychoanaleptics, and $41 \%$ of subjects with unknown diagnoses took antithrombotic agents.

\section{Clinical Trials Safety Results}

Among 1,180 safety evaluable subjects in the 10 completed clinical trials, 643 AEs were reported. The number of subjects with at least $1 \mathrm{AE}$ was $261(22 \%)$.

The number of subjects with at least $1 \mathrm{AE}$ leading to discontinuation from study was $14(1 \%)$, leading to death was 5 $(<1 \%)$, and considered a serious AE was $44(4 \%)$. 
The MedDRA preferred term for a given $\mathrm{AE}$ is reported throughout these results, unless otherwise specified. Overall (Table 2), the most frequently occurring AEs ( $\geq 1 \%$ of all subjects) included headache (42 subjects [4\%]), dizziness (21 subjects [2\%]), nausea (21 subjects [2\%]), nasopharyngitis (16 subjects [1\%]), and injection site hematoma (12 subjects [1\%]). AEs occurring in more than 1 subject are listed in Supplemental Table 5.

For most subjects with an AE (218/1,180 [19\%]), the maximum intensity was mild (124 [11\%]) or moderate (94 [8\%]) (Table 2). Thirty-nine subjects $(3 \%)$ had AEs that were severe in intensity, only one of which (headache) was considered related to DaTscan. Intensity data were missing from 4 subjects. The following severe AEs occurred in more than 1 subject (data not shown): fall (4 subjects $[<1 \%]$ ); femoral neck fracture (3 subjects); and abdominal pain, pneumonia, joint dislocation, and dementia (2 subjects each).

Forty-two subjects $(4 \%)$ had an $\mathrm{AE}$ considered by the investigator to be at least possibly related to DaTscan or its administration (Table 2). Among these, the following AEs were reported in more than 1 subject (data not shown): headache (14 subjects $[1 \%]$ ); nausea (8 subjects $[<1 \%]$ ); vertigo, dry mouth, hunger, injection site hematoma, dizziness, and formication (i.e., paraesthesia) (3 subjects each); and balance disorder and dysgeusia (2 subjects each).

A comparison of sex and age subgroups among subjects with at least $1 \mathrm{AE}$ is shown in Figure 2. A larger absolute percentage of subjects who were female $(24 \%$ vs. $21 \%),<65$ y old $(25 \%$ vs. $20 \%$ ), and $<75$ y old ( $25 \%$ vs. $16 \%$ ) experienced at least $1 \mathrm{AE}$.

All of the AEs leading to discontinuation of 14 subjects from the study for safety reasons were considered by the investigator to be not related to DaTscan except for 1 (limbic encephalitis, occurring $81 \mathrm{~d}$ after DaTscan dose) considered unlikely related.

TABLE 2

AEs in 10 Completed DaTscan Clinical Trials

\begin{tabular}{|c|c|c|c|c|}
\hline \multirow[b]{2}{*}{$\mathrm{AE}$} & \multicolumn{2}{|c|}{ All } & \multicolumn{2}{|c|}{ Related } \\
\hline & $n$ & $\%$ & $n$ & $\%$ \\
\hline No. of AEs & 643 & & 76 & \\
\hline No. of subjects with any $\mathrm{AE}$ & 261 & 22 & 42 & 4 \\
\hline \multicolumn{5}{|l|}{ AEs by maximum intensity } \\
\hline Mild & 124 & 11 & 26 & 2 \\
\hline Moderate & 94 & 8 & 15 & 1 \\
\hline Severe & 39 & 3 & 1 & $<1$ \\
\hline \multicolumn{5}{|l|}{ AEs in $\geq 1 \%$ of all subjects } \\
\hline Headache & 42 & 4 & 14 & 1 \\
\hline Nausea & 21 & 2 & 8 & $<1$ \\
\hline Dizziness & 21 & 2 & 3 & $<1$ \\
\hline Nasopharyngitis & 16 & 1 & 0 & 0 \\
\hline Injection site hematoma & 12 & 1 & 3 & $<1$ \\
\hline No. of subjects with any SAE & 44 & 4 & 0 & 0 \\
\hline \multicolumn{5}{|l|}{ SAEs in $>1$ subject } \\
\hline Chest pain & 3 & $<1$ & 0 & 0 \\
\hline Fall & 3 & $<1$ & 0 & 0 \\
\hline Femoral neck fracture & 3 & $<1$ & 0 & 0 \\
\hline Abdominal pain & 2 & $<1$ & 0 & 0 \\
\hline Pneumonia & 2 & $<1$ & 0 & 0 \\
\hline Joint dislocation & 2 & $<1$ & 0 & 0 \\
\hline Dementia & 2 & $<1$ & 0 & 0 \\
\hline $\begin{array}{l}\mathrm{SAE}=\text { serious } \mathrm{AE} . \\
n=1,180 \text { subjects. }\end{array}$ & & & & \\
\hline
\end{tabular}

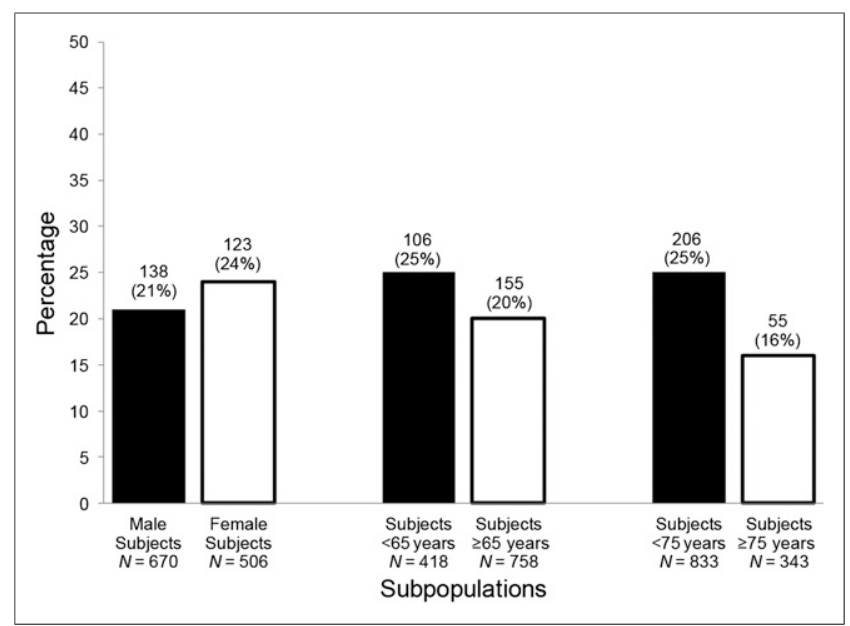

FIGURE 2. Percentages of subjects with $1 \mathrm{AE}$ or more, by subgroup (age categories and sex).

For 4 subjects in PDT304, the discontinuation events were fatal. The fatal events (verbatim term) were hospitalization due to bronchial carcinoma (466 d postdose [after 1 dose]); pneumonia (899 d postdose [after 2 doses]); septicemia (onset unavailable); and fractured left femoral neck, left ventricular failure, and ischemic heart disease ( 3 events in 1 subject: 219, 224, and 225 d postdose, respectively [after 1 dose]). A fifth death, which occurred in PDT301, was due to a femoral neck fracture (onset 3 d postdose) for which surgery was performed; postoperatively, the subject contracted Clostridium difficile and suffered subsequent complications leading to death.

The nonfatal AEs leading to discontinuation were surgery for excision of bronchial neoplasm (633 d postdose [after 1 dose]), hospitalization for suspected cardiovascular accident $(1,038 \mathrm{~d}$ postdose [after 2 doses]), non-Hodgkin lymphoma, ( $\sim 762 \mathrm{~d}$ postdose [after 2 doses]), pancreatitis (onset unavailable), double kidney transplant (967 d postdose [after 2 doses]), gastric cancer stage 0 ( $\sim 973 \mathrm{~d}$ post dose [after 2 doses]), hospitalization due to excess opiates (onset unavailable), exacerbation of osteoarthritis ( $151 \mathrm{~d}$ postdose), and the case of limbic encephalitis ( $81 \mathrm{~d}$ postdose) mentioned above. The latter 3 events occurred in GE-001-013; all other events leading to discontinuation occurred in PDT304.

Among the 44 subjects with at least 1 serious AE (Table 2), the following serious AEs occurred in more than 1 subject: chest pain, fall, and femoral neck fracture $(3$ subjects each $[<1 \%])$ and abdominal pain, pneumonia, joint dislocation, and dementia (2 subjects each). In PDT304, 1 subject underwent prostatectomy for cancer $627 \mathrm{~d}$ after the first dose and went on to receive the second and third doses uneventfully. None of the serious AEs, including the AEs that led to death, was considered by the investigator to be at least possibly related to DaTscan.

The AE profiles for subjects receiving more than 1 dose of DaTscan were similar to the overall profile (which includes these 172 subjects).

Subjects receiving 2 doses of DaTscan came from studies PDT304 $(n=127)$, PDT03007 $(n=31)$, and PDT408 $(n=14)$. In PDT304, 151 AEs were reported after the second dose (but before the third dose) of DaTscan; the most frequent AEs were headache (10 events), fall (5 events), and depression (4 events); subjects who died or withdrew were described above. In PDT03007, only 8 AEs were reported after the second dose; the 
most frequent events were dizziness ( 3 events) and headache (2 events). In PDT408, no AEs were reported after the second dose.

All 99 subjects receiving 3 doses of DaTscan came from PDT304. Nineteen AEs were reported after the third dose; the most frequent AEs were nasopharyngitis (3 events) and dizziness (2 events).

No AEs consistent with cocaine effects were observed. There were no laboratory, vital signs, or electrocardiogram results of clinical concern.

\section{Postapproval Safety Results}

On the basis of 518,419 single-dose vials dispatched (24,281 [4.7\%] in the United States and the rest in the EU), and allowing for wastage and nonclinical use, an estimated 516,000 additional patients were exposed to DaTscan from July 2000 through July 2013. One death was reported. In an investigator-initiated interventional trial of $\mathrm{AD}, 1$ patient had a hemorrhagic stroke $17 \mathrm{~d}$ after receiving DaTscan, which was reported by the investigator to be unrelated to DaTscan; the patient died 3 d later. Two additional serious spontaneously reported ADRs after the use of DaTscan were submitted: epileptic seizure and blood sodium decreased (Table 3). In both cases, a causal relationship with DaTscan was not considered to be a reasonable possibility. Thirty-two spontaneous nonserious ADRs were also submitted. Of these, injection site pain or irritation ( 8 cases, 4 of which were reported by the same hospital in an 8-d period), hypersensitivity (4 cases), headache (3 cases), nausea (2 cases), and dysgeusia (1 case) were reactions listed in the label. Unlisted, nonserious reactions were as follows: altered biodistribution (6 cases); syncope ( 2 cases); and single cases of anxiety, sense of oppression, facial

TABLE 3

Adverse Drug Experiences (All Spontaneous Safety Reports)

\begin{tabular}{|c|c|c|c|}
\hline $\mathrm{AE}$ & Listed* & Unlisted & Total \\
\hline No. of serious events & 0 & 2 & 2 \\
\hline \multicolumn{4}{|l|}{ Serious events } \\
\hline Blood sodium decreased & & $x$ & 1 \\
\hline Epilepsy & & $x$ & 1 \\
\hline No. of nonserious events & 18 & 14 & 32 \\
\hline \multicolumn{4}{|l|}{ Nonserious events } \\
\hline Injection site pain ${ }^{\dagger}$ & $x$ & & 8 \\
\hline Altered biodistribution & & $x$ & 6 \\
\hline Hypersensitivity ${ }^{\ddagger}$ & $x$ & & 4 \\
\hline Headache & $x$ & & 3 \\
\hline Nausea $^{\ddagger}$ & $x$ & & 2 \\
\hline Syncope & & $x$ & 2 \\
\hline Anxiety & & $x$ & 1 \\
\hline Cold sweat & & $x$ & 1 \\
\hline Dysgeusia $¥$ & $x$ & & 1 \\
\hline Epistaxis & & $x$ & 1 \\
\hline Facial pain & & $x$ & 1 \\
\hline Influenzalike illness & & $x$ & 1 \\
\hline Sense of oppression & & $x$ & 1 \\
\hline
\end{tabular}

*Included in current reference safety information (listed)-that is, European Summary of Product Characteristics (March 2013) or U.S. prescribing information (April 2011).

${ }^{\dagger}$ Added in 2002.

${ }^{\ddagger}$ Added in 2011.

Approximately half a million doses of DaTscan dispensed in 13-y period. $\mathrm{x}$ indicates whether event is currently mentioned in reference safety information (listed) or not (unlisted). pain, influenzalike illness, epistaxis, and cold sweat. The altered biodistribution events (unexpected areas of enhancement or lack of expected enhancement) were more of an efficacy, rather than a safety, issue. Two of these cases were potentially due to medications (methylphenidate and sertraline) listed in the label as interacting drugs.

There were no spontaneous reports regarding overdose, drug abuse or misuse, experience during pregnancy or lactation, or experience in special patient groups (e.g., the elderly, renal or hepatic impairment).

\section{DISCUSSION}

Because all 10 clinical trials were open-label, individual investigator reporting of AEs in the clinical trials component of the analysis may potentially reflect subjective bias, as in any openlabel investigation. However, the (regulatory) definition of $\mathrm{AE}$ and serious AE were the same across studies. Our outcome selection for reporting was conservative (drilled down to the level of reporting of MedDRA preferred terms-i.e., AEs, reported for 2 or more subjects among 1,180 subjects). Safety in clinical trials for this radiodiagnostic was monitored with rigor comparable to that for investigational new drugs.

AEs were reported in the 10 completed clinical trials with relatively low frequency $(22 \%)$, and most were mild $(11 \%)$ to moderate $(8 \%)$. Few $(4 \%)$ were considered to be at least possibly related to DaTscan or its administration by expert clinicians.

Sex- and age-related differences in DaT density in HV (14-17) and PD $(17,18)$ have been reported, though the differences in frequency of AEs between the sex and age subgroups did not appear to be clinically meaningful; no specific events occurred with greater frequency in the older subgroups than in the general pool. Overall thyroid blockade was implemented $84 \%$ of the time. For the $8 \mathrm{NDA}$ trials, thyroid-blockade procedures were implemented $94 \%$ of the time. In the phase- 4 trials, thyroid blockade was to be performed according to hospital routine (i.e., was not protocol-specified), which would be expected to reduce its implementation but might also be less uniformly recorded. Common side effects of thyroid-blocking medication (potassium iodide) may included acne, loss of appetite, upset stomach, fever, weakness, unusual tiredness, swelling in the neck or throat, mouth sores, skin rash, nausea, vomiting, stomach pains, irregular heartbeat, numbness or tingling of the hands or feet, or a metallic taste in the mouth. We observed some of these typical side effects (among them nausea occurred with the greatest frequency [2\%], and fatigue, lethargy, vomiting, abdominal pain, paraesthesia, dysgeusia, rash, arrhythmia, and atrial fibrillation, each $\leq 0.3 \%$ ). No issues were identified among the other (objective) measurements of safety parameters (laboratory, vital signs, or electrocardiogram) in the clinical trials, so other safety variables are not summarized here but are summarized through mid-2009 elsewhere (10).

ADRs reported were rare; 34 ADRs were reported for an estimated 516,000 patients receiving DaTscan, only 2 of which were serious (epilepsy and hyponatremia) and 1 of which (stroke) resulted in death (all 3 unrelated to DaTscan). Though deemed uncommon (frequency $\geq 1 / 1,000$ to $<1 / 100$ ), injection site pain (intense pain after administration into small veins) warrants mention. The label has been updated since licensure to reflect that a slow intravenous injection (not $<15-20 \mathrm{~s}$ ) via an arm vein is recommended. 
The 2 cases of altered biodistribution serve to illustrate the importance of ensuring that drugs that may interact with DaTscan are washed out before scanning to enable an accurate read, the relevant safety point being, of course, unnecessary irradiation. To expound on concurrent medications, during clinical development it was established that levodopa has no effect on DaTscan images assessed quantitatively (19). The same is true of other dopamine agonists, for example, pramipexole (in nigral-lesioned mice) and pergolide $(20,21)$ when visualized with SPECT using ${ }^{123}$ I- $\beta$-CIT (a radiodiagnostic of similar chemical structure and mechanism of action as DaTscan) (22), and likely true of ropinirole (which, like pramipexole, is a D2/D3 agonist with similar mechanism of action) $(23,24)$. Conveniently, these medications need not be washed out. A published review also concluded that dopaminergic drugs do not influence the visual interpretation of DaTscan images (25). DaTscan does have some affinity for the serotonin transporter. Selective serotonin reuptake inhibitors have been shown to increase the specific striatal-to-nonspecific binding of DaTscan from $10 \%$ (paroxetine) (26) to $18 \%$ (citalopram) (27); the effect is homogeneous, and in the experience of 1 author, this does not represent a clinical problem. However, dopaminergic central nervous system stimulants are known to have a significant influence and therefore should be stopped before the scan (28).

All 10 trials and label recommendations specified a comparable DaTscan administration procedure using a dose of 111-185 MBq, preceded by thyroid blockade, and followed by a saline flush. Regulatory requirements for reporting clinical trials safety data in applications for licensure are comprehensive, and we adhered to both regulatory and ICH standards for both preapproval and postapproval safety reporting.

The published literature on DaTscan is overwhelmingly efficacy-related; mention of safety gleaned from the literature, although limited, is consistent with what is reported here (e.g., $6,29,30)$ and listed in the label $(2,3)$.

Our clinical observations and conclusions are consistent extensions of observations made during preclinical safety investigations of nonradioactive ioflupane (preclinical data from DaTscan NDA). We observed no clinical effect on blood pressure, just as none was observed preclinically (at 10 times multiples of exposure by comparison); and, we observed a low rate of cancer diagnosis in this elderly population (2.8\% in PDT304, the 3-y, 3dose study), just as the nonclinical evidence suggests ioflupane is not genotoxic (at 1,000 times multiples of exposure by comparison). For reference, according to the National Cancer Institute's Surveillance Epidemiology and End Results 1975-2010 data, the incidence of all cancers in both sexes, with no adjustment for concurrent illness, in persons aged 65 y and older was $2.1 \%$ (31).

Ioflupane is rapidly extracted from the blood into organs. In human plasma after intravenous administration, the amount of ${ }^{123}$ I-ioflupane declined to about $40 \%$ of the total plasma radioactivity at $2 \mathrm{~h}$ and $20 \%$ at $4 \mathrm{~h}$ (32). Similarly, none of its radiometabolites appears in plasma (or is sufficiently lipophilic to cross the blood-brain barrier) in significant quantities $(32,33)$. Ioflupane is excreted through both urinary and hepatobiliary routes. At $48 \mathrm{~h}$ after intravenous injection, mean measured urine excretion $( \pm \mathrm{SD})$ was $60 \% \pm 9 \%$, and mean predicted fecal excretion was $14 \% \pm$ $1 \%$ (34). In general, the striatum received the highest absorbed dose (average, $0.23 \mathrm{mGy} / \mathrm{MBq}$ ), followed by the urinary bladder wall (average, $0.054 \mathrm{mGy} / \mathrm{MBq}$ ) and lungs (average, $0.043 \mathrm{mGy} /$ MBq) (34). The physical half-life of ${ }^{123}$ I is $13.2 \mathrm{~h}$. Few events associated with these biokinetic properties occurred. Although subjects with mild-to-moderate renal and hepatic impairment were allowed in the studies (and the DaTscan label does not recommended use in persons with significant renal or hepatic impairment), there was not any specific abnormal renal or hepatic laboratory value reported as an $\mathrm{AE}$ in more than 2 subjects. The only system organ class with greater than $1 \%$ of subjects for whom AEs were considered by the expert clinician to be related to DaTscan was nervous system disorders (2\%), half of which were headache $(1 \%)$, and the next most frequent were dizziness and paraesthesia (3 subjects each).

For DaTscan, the whole-body effective dose resulting from an administered activity of $185 \mathrm{MBq}(5 \mathrm{mCi})$ is $3.94 \mathrm{mSv}$ in an adult (2), less than that for a CT scan $(10 \mathrm{mSv})$. For reference, in the United States, the dose of ionizing radiation per person from nonmedical sources is approximately $3-4 \mathrm{mSv} / \mathrm{y}$ (35). For an adult examination, even a $12-\mathrm{mSv}$ dose is considered low, defined as an added lifetime risk of fatal cancer of $0.01 \%-0.1 \%$ (36). For reference, in the United States the lifetime risk of fatal cancer is 1 in 5 (31).

Although we certainly do not advocate unnecessary scans, the radiation-associated risk must be weighed against the usefulness of DaTscan imaging. Most clinicians recognize intuitively that assessing theoretic risk is most meaningful when considered in the context of potential benefit.

Accordingly, DaTscan is approved for use in very specific circumstances - that is, where the benefit of an accurate diagnosis of PS versus ET (in the United States and EU) or additionally AD versus DLB (in the EU) is likely to outweigh the risk of minimal exposure to radioactivity to help avoid side effects of inappropriate medications or an unnecessary delay in instituting appropriate therapy to alleviate disability (5).

Other than modest revision of the reference safety information (addition of headache, increased appetite, paraesthesia, vertigo, injection site pain, nausea, and dysgeusia), no regulatory authority or clinical trial sponsor action has been needed since first approval in 2000. On the contrary, therapeutic indications have been expanded in Europe to include DLB versus AD (in 2006) and early PS (in 2013). As we learn more about it (17,37), DaTscan SPECT appears to be becoming more useful; under indicated circumstances, it is recommended by the European Federation of Neurologic Societies, Movement Disorders Society-EU Section, and European Neurologic Society (38-40), the Society of Nuclear Medicine and Molecular Imaging (29), and the U.K. National Institute for Health and Clinical Excellence (41).

\section{CONCLUSION}

Comprehensive analysis of DaTscan safety data, both from clinical trials database and postapproval surveillance perspectives, showed that AEs and adverse reactions, respectively, associated with the diagnostic radiopharmaceutical DaTscan have been infrequent and predominately mild. DaTscan was well tolerated. No serious AE or spontaneously reported serious ADR was likely to be related to DaTscan or its administration. No unlisted, nonserious reaction reported postapproval was considered to be a safety concern.

\section{DISCLOSURE}

The costs of publication of this article were defrayed in part by the payment of page charges. Therefore, and solely to indicate this fact, this article is hereby marked "advertisement" in accordance 
with 18 USC section 1734. This report and associated clinical trials were sponsored by GE Healthcare, Princeton, New Jersey. Additional details of disclosure are presented online in the Supplemental Disclosure Appendix. No other potential conflict of interest relevant to this article was reported.

\section{ACKNOWLEDGMENTS}

We thank all the study subjects and their relatives for participating in the studies; the more than 200 coinvestigators (neurologists and nuclear medicine specialists listed in the Supplemental Coinvestigator Appendix) and their staff; and GE Healthcare operational and supporting staff. We are grateful to Dr. Emilio Moreno (GE Healthcare, Spain) for reviewing the manuscript, and Duncan Lane (GE Healthcare) for providing details from the GE Healthcare clinical development safety database. Independent consultants Earl Webb Henry, MD (for statistical analysis and comments), and Carlyn K. Rosenberg, MS (for writing and editorial assistance), both supported indirectly by GE Healthcare, are gratefully acknowledged.

\section{REFERENCES}

1. Fowler JS, Volkow ND, Wang G, Gatley SJ, Logan J. [11]Cocaine: PET studies of cocaine pharmacokinetics, dopamine transporter availability and dopamine transporter occupancy. Nucl Med Biol. 2001;28:561-572.

2. DaTscan ${ }^{\mathrm{TM}}$ [US package insert]. Arlington Heights, IL: GE Healthcare, MediPhysics Inc.; 2011.

3. European Medicines Agency. DaTSCAN ${ }^{\mathrm{TM}}$ Summary of Product Characteristics. http://www.ema.europa.eu/ema/index.jsp?curl=pages/medicines/human/medicines/ 000266/human_med_000739.jsp\&mid=WC0b01ac058001d124. Accessed May 21, 2014.

4. Walker Z, Jaros E, Walker RW, et al. Dementia with Lewy bodies: a comparison of clinical diagnosis, FP-CIT single photon emission computed tomography imaging and autopsy. J Neurol Neurosurg Psychiatry. 2007;78:1176-1181.

5. Bajaj N, Hauser RA, Grachev ID. Clinical utility of dopamine transporter single photon emission CT (DaT-SPECT) with $\left({ }^{123} \mathrm{I}\right)$ ioflupane in diagnosis of parkinsonian syndromes. J Neurol Neurosurg Psychiatry. 2013;84:1288-1295.

6. Kupsch AR, Bajaj N, Weiland F, et al. Impact of DaTscan SPECT imaging on clinical management, diagnosis, confidence of diagnosis, quality of life, health resource use and safety in patients with clinically uncertain parkinsonian syndromes: a prospective 1-year follow-up of an open-label controlled study. $J$ Neurol Neurosurg Psychiatry. 2012;83:620-628.

7. Walker Z, McKeith I, Rodda J, et al. Comparison of cognitive decline between dementia with Lewy bodies and Alzheimer's disease: a cohort study. BMJ Open. 2012;2:e000380.

8. Hauser RA, Grosset DG. [ ${ }^{123}$ I]FP-CIT (DaTscan) SPECT brain imaging in patients with suspected parkinsonian syndromes. J Neuroimaging. 2012;22:225-230.

9. Benamer HTS, Oertel WH, Patterson J, et al. Prospective study of presynaptic dopaminergic imaging in patients with mild parkinsonism and tremor disorders: part 1. Baseline and 3-month observations. Mov Disord. 2003;18:977-984.

10. U.S. FDA. PCNS Advisory Committee Briefing Document. http://www.fda.gov/ downloads/AdvisoryCommittees/UCM176192. Accessed May 21, 2014.

11. MedDRA 11.0 Review. Meddrahelp.com. http://www.meddrahelp.com/PDF/ MedDRA_11_0_Review.pdf. Accessed June 10, 2014.

12. MedDRA 13.1 Review. Meddrahelp.com. http://www.meddrahelp.com/PDF/ MedDRA_13_1.pdf. Accessed June 10, 2014.

13. MedDRA 15.0 Review. Meddrahelp.com. http://www.meddrahelp.com/pdf/ MedDRA_15_0.pdf. Accessed June 10, 2014.

14. van Dyck CH, Seibyl JP, Malison RT, et al. Age-related decline in dopamine transporters: analysis of striatal subregions, nonlinear effects, and hemispheric asymmetries. Am J Geriatr Psychiatry. 2002;10:36-43.

15. Varrone A, Dickson JC, Tossici-Bolt L, et al. European multicentre database of healthy controls for $\left[{ }^{123} \mathrm{I}\right] \mathrm{FP}-\mathrm{CIT}$ SPECT (ENC-DAT): age-related effects, gender differences and evaluation of different methods of analysis. Eur J Nucl Med Mol Imaging. 2013;40:213-227.

16. Eusebio A, Azulay J, Ceccaldi M, Girard N, Mundler O, Guedj E. Voxel-based analysis of whole-brain effects of age and gender on dopamine transporter SPECT imaging in healthy subjects. Eur J Nucl Med Mol Imaging. 2012;39:1778-1783.
17. Seibyl J, Jennings D, Grachev I, Coffey C, Marek K. 123-I Ioflupane SPECT measures of Parkinson disease progression in the Parkinson Progression Marker Initiative (PPMI) trial. J Nucl Med. 2013;54(suppl 2):57-58.

18. Schapira AH, McDermott MP, Barone P, et al. Pramipexole in patients with early Parkinson's disease (PROUD): a randomised delayed-start trial. Lancet Neurol. 2013;12:747-755.

19. Schillaci O, Pierantozzi M, Filippi L, et al. The effect of levodopa therapy on dopamine transporter SPECT imaging with ${ }^{123}$ I-FP-CIT in patients with Parkinson's disease. Eur J Nucl Med Mol Imaging. 2005;32:1452-1456.

20. Depboylu C, Maurer L, Matusch A, et al. Effect of long-term treatment with pramipexole or levodopa on presynaptic markers assessed by longitudinal $\left[{ }^{123} \mathrm{I}\right]$ FP-CIT SPECT and histochemistry. Neuroimage. 2013;79:191-200.

21. Winogrodzka A, Bergmans P, Booij J, van Royen EA, Stoof JC, Wolters EC. $\left[{ }^{123} \mathrm{I}\right] \beta$-CIT SPECT is a useful method for monitoring dopaminergic degeneration in early stage Parkinson's disease. J Neurol Neurosurg Psychiatry. 2003;74: 294-298.

22. Seibyl JP, Marek K, Sheff K, et al. Iodine-123- $\beta$ CIT and iodine-123-FPCIT SPECT measurement of dopamine transporters in healthy subjects and Parkinson's patients. J Nucl Med. 1998;39:1500-1508.

23. Mirapex ${ }^{\circledR}$ (pramipexole) [US package insert]. Ridgefield, CT: Boehringer Ingelheim; 2013.

24. Requip (ropinirole) XL [US package insert]. Research Triangle Park, NC: GlaxoSmithKline; 2009.

25. Booij J, Kemp P. Dopamine transporter imaging with $\left[{ }^{123} \mathrm{I}\right]$ FP-CIT SPECT: potential effects of drugs. Eur J Nucl Med Mol Imaging. 2008;35:424-438.

26. Booij J, de Jong J, de Bruin K, Knol R, de Win MM, van Eck-Smit BL. Quantification of striatal dopamine transporters with ${ }^{123}$ I-FP-CIT SPECT is influenced by the selective serotonin reuptake inhibitor paroxetine: a double-blind, placebocontrolled, crossover study in healthy control subjects. J Nucl Med. 2007;48: 359-366.

27. Ziebell M, Holm-Hansen S, Thomsen G, et al. Serotonin transporters in dopamine transporter imaging: a head-to-head comparison of dopamine transporter SPECT radioligands ${ }^{123}$ I-FP-CIT and ${ }^{123}$ I-PE21. J Nucl Med. 2010;51:18851891.

28. Kägi G, Bhatia KP, Tolosa E. The role of DAT-SPECT in movement disorders. J Neurol Neurosurg Psychiatry. 2010;81:5-12.

29. Djang DS, Janssen MJR, Bohnen N, et al. SNM practice guideline for dopamine transporter imaging with ${ }^{123}$ I-ioflupane SPECT 1.0. J Nucl Med. 2012;53:154163.

30. Mouraeff Y, Farid K, Poullias X, Goncalves A, Petras S, Caillat-Vigneron N. Thyroid protection before ${ }^{123} \mathrm{I}$-ioflupane (DaTSCAN) imaging. Eur J Nucl Med Mol Imaging. 2011;38:786-787.

31. Howlader N, Noone AM, Krapcho M, et al., eds. SEER Cancer Statistics Review, 1975-2010, National Cancer Institute. http://seer.cancer.gov/csr/1975_2010/. 2013. Accessed May 21, 2014.

32. Bergstrom KA, Halldin C, Lundkvist C, et al. Characterisation of C-11 and I-123 labelled $\beta$-CIT-FP and $\beta$-CIT-FE metabolism measured in monkey and human plasma: identification of two labelled metabolites with HPLC. Hum Psychopharmacol. 1996;11:483-490.

33. Tanaka A, Okano K, Tamagami H, et al. Metabolism of ${ }^{123}$ I-FP-CIT in humans. Kaku Igaku. 1999;36:745-751.

34. Booij J, Busemann Sokole E, Stabin MG, Janssen AG, de Bruin K, van Royen EA. Human biodistribution and dosimetry of $\left[{ }^{123} \mathrm{I}\right]$ FP-CIT: a potent radioligand for imaging of dopamine transporters. Eur J Nucl Med. 1998;25:24-30.

35. American Nuclear Society. Radiation Dose Chart. http://www.ans.org/pi/resources/ dosechart/. Accessed June 10, 2014.

36. American College of Radiology and Radiological Society of North America. Patient Safety: Radiation Dose in X-Ray and CT Exams. RadiologyInfo.org. http:// www.radiologyinfo.org/en/safety/index.cfm?pg=sfty_xray. Accessed May 21, 2014.

37. Söderlund TA, Dickson JC, Prvulovich E, et al. Value of semiquantitative analysis for clinical reporting of ${ }^{123} \mathrm{I}-2-\beta$-carbomethoxy-3 $\beta$-(4-iodophenyl)- $N$-(3fluoropropyl)nortropane SPECT studies. J Nucl Med. 2013;54:714-722.

38. Sorbi S, Hort J, Erkinjuntti T, et al. EFNS-ENS guidelines on the diagnosis and management of disorders associated with dementia. Eur J Neurol. 2012;19: 1159-1179.

39. Filippi M, Agosta F, Barkhot F, et al. EFNS task force: the use of neuroimaging in the diagnosis of dementia. Eur J Neurol. 2012;19:e131-e140.

40. Berardelli A, Wenning GK, Antonini A, et al. EFNS/MDS-ES/ENS [corrected] recommendations for the diagnosis of Parkinson's disease. Eur J Neurol. 2013; 20:16-41.

41. National Institute for Health and Clinical Excellence (NICE). NICE Clinical Guideline 35: Parkinson's disease: diagnosis and management in primary and secondary care. Nice.org. http://www.nice.org.uk/nicemedia/live/10984/30088/ 30088.pdf. June 2006. Accessed May 21, 2014. 